\title{
Designer Scaffolds for Bioengineered Tissue
}

\section{Interfaces}

Ryan J. Hickey ${ }^{1}$, Maxime Leblanc Latour ${ }^{1}$, James L. Harden ${ }^{1,2}$, and Andrew E. Pelling ${ }^{1,3-5, *}$

${ }^{1}$ Department of Physics, STEM Complex, 150 Louis Pasteur Pvt, University of Ottawa, Ottawa, ON, K1N6N5 Canada

${ }^{2}$ Ottawa Institute of Systems Biology, University of Ottawa, Ottawa, ON, K1H8M5 Canada

${ }^{3}$ Department of Biology, Gendron Hall, 30 Marie Curie, University of Ottawa, Ottawa, ON, K1N5N5 Canada

${ }^{4}$ Institute for Science Society and Policy, Simard Hall, 60 University, University of Ottawa, Ottawa, ON, K1N5N5 Canada

${ }^{5}$ SymbioticA, School of Anatomy, Physiology and Human Biology, University of Western Australia, Perth, WA, 6009

* Author for correspondence

Prof. Andrew E. Pelling, Email: a @ pellinglab.net 


\begin{abstract}
In regenerative medicine, the healing of the interfacial zone between tissues is a major challenge, yet approaches for engineering and studying the complex microenvironment of this interface remain lacking ${ }^{1-3}$. Here, we create and study these complex living interfaces by manufacturing modular "blocks" of decellularized plant-derived scaffolds ${ }^{4-6}$ with varying shapes and sizes with a computer numerical controlled mill. Each block can then be seeded with different cell types and easily assembled in a manner akin to $\mathrm{LEGO}^{\mathrm{TM}}$ bricks to create an engineered tissue interface (ETI). As a proof-of-concept study we utilize ETIs to investigate the interaction between lab grown bone and connective tissues. We also demonstrate how ETIs are biocompatible in vivo, stimulating the formation of blood vessels, cell infiltration, and tissue integration after implantation. This work creates possibilities for new tissue design avenues for understanding fundamental biological processes or the development of synthetic artificial tissues.
\end{abstract}

\title{
INTRODUCTION
}

The body consists of a variety of tissues, and the interface between them presents a unique juxtaposition of interacting cellular environments ${ }^{7-9}$. Traditionally, bioengineers design scaffolds for one environment, but there is a need to develop model systems to create and investigate tissue interfaces $^{10-14}$. We constructed living engineered tissue interfaces (ETIs) by combining highly porous, decellularized, plant-derived scaffolds in a modular fashion. Plant-derived scaffolds can be selected to mimic natural human structures in vitro and in vivo ${ }^{4-6,15-23}$. Intense research has been focused on designing biomaterials to enhance tissue integration in specific cases ${ }^{24-27}$; however, the widespread potential of this technology necessitates a platform that can be adapted to suit particular biomedical needs. We hypothesized that the modular assembly of biomaterial 
subunits could create ETIs. We found that combining scaffolds in a LEGO ${ }^{\mathrm{TM}}$-like fashion is a viable approach for establishing an ETI platform.

The physical environment affects many cellular processes including differentiation, migration, force generation, proliferation, spreading, alignment, and gene expression ${ }^{28-31}$. The interplay between the physical characteristics of the microenvironment and biochemical signaling is complex, and extensive research has been dedicated to elucidating the mechanisms responsible for these phenomena ${ }^{32-36}$. These insights have highlighted a need to develop strategies for the fabrication of biomimetic constructs that more closely match the mechanical properties of the native environment of the cell ${ }^{37}$.

Another layer of complexity is added when considering the physical interface between tissues. These regions often bridge at least two biophysically distinct environments, and the transitional zone has its own multifaceted organization. For example, tissue entheses contain graded insertion sites with uncalcified and calcified regions, different extracellular matrix proteins (e.g. collagen type), and specific fibrillar arrangements in order to create a graduated transition between stiff and soft tissues; these interfacial zones are susceptible to injury and are difficult to repair and regenerate ${ }^{1-3,7}$. Although damage to the interfaces between distinct tissues and structures within the body can have significant consequences, there are few, if any, bioengineering strategies to study their regeneration from the perspective of basic fundamental science or clinical translation. Therefore, development of novel strategies to biofabricate artificial tissue interfaces may lend novel insights into how such regions may be designed, engineered, and repaired in a regenerative medicine context.

Moreover, when attempting to create designer biomaterials and scaffolds for fabricating tissue interfaces it is important to also consider the mechanical performance and matching of 
physical properties with the surrounding environment. Such considerations are integral for effective biomaterial design and performance ${ }^{38-40}$ as mechanical/physical mismatch can lead to poor tissue integration which is ultimately a leading cause of implant failure ${ }^{2}$. Additionally, the transitional zones between tissues are exposed to a wide variety of mechanical forces ${ }^{41-44}$. Tools to study the responses to stresses and strains applied to these interfaces are required to advance our understanding of how these regions respond to compression, tension, and shear. Designer scaffolds for artificial tissue interfaces can help tease apart the gaps in knowledge surrounding tissue interfaces. Growing two or more distinct tissues and bringing them together into one functional unit as an ETI presents exciting possibilities to design artificial tissues that have analogues in the human body, but going a step further, engineering tissues that have unique physical properties.

Our previous work reveals that the decellularized plant-derived scaffolds have a Young's modulus that is comparable to that of various human tissues and cell types; moreover, specific plant candidates can be chosen to mimic particular structures in the body. Nevertheless, simple compression measurements fail to fully characterize the mechanical properties of these scaffolds. In the body, our tissues are subjected to complex shear forces in addition to simple tensile and compressive loads. Here, we provide a more thorough mechanical characterization of the ETI. These materials are viscoelastic materials that behave largely as elastic bodies. This platform for ETI design establishes a "plug and play" method for combining multiple cell types and distinct physical environments. 


\section{RESULTS}

\section{Engineered Tissue Interface (ETI) Fabrication}

In order to create ETIs, we combined subunit components without the use of additional crosslinkers or glues. Interlocking composite pieces were fabricated to yield scaffolds that could be repopulated with distinct or identical cell types and subsequently re-combined into a single unit (Fig. 1) ${ }^{6}$. To create such scaffolds, we employed 3D milling with a computer numerical controlled (CNC) router to reproducibly carve the structures from apple hypanthium tissue (Fig. 1a, Supplementary Video 1). A simple design for interlocking materials is the common stud and antistud geometry of LEGO ${ }^{\text {TM }}$ bricks (Fig. 1b and Supplementary Fig. 1). We designed single stud and anti-stud bricks, wherein the stud subunit consisted of a $5 \mathrm{~mm}$ x $5 \mathrm{~mm}$ x $2 \mathrm{~mm}$ base with a $2 \mathrm{~mm}$ (height and diameter) cylindrical peg protruding from the center, and the anti-stud subunit had a 2 $\mathrm{mm}$ diameter cylindrical hole in the center of the $5 \mathrm{~mm}$ x $5 \mathrm{~mm}$ × $2 \mathrm{~mm}$ base (Fig. 1b). Tissue interface formation was a direct consequence of interlocking the components together (Fig. 1c). Prior to assembly, the individual subunits were decellularized, processed, and repopulated with mammalian cells as previously described ${ }^{6}$ (Fig. 1d and Supplementary Fig. 2).

\section{Cell Migration}

Cells must be able to traverse the interface and interact with the adjacent population when required $^{45}$. Controlled in vitro cell migration assays were carried out, which revealed that when a scaffold pre-loaded with fibroblast cells was interlocked with a bare subunit, the cells migrated across the interface and invaded the bare scaffold (Fig. 1e). The proliferation was monitored 
weekly for 3 weeks, and each week presented a significant increase in cell infiltration $(\mathrm{p}<0.001)$

(Fig. 1e, g). After 3 weeks of culture, the initially barren scaffold had a comparable cell number density to the pre-seeded subunit (Fig. 1e, g).

We repeated the cell migration assays with fibroblasts loaded on both subunits. The two fibroblast groups were distinguishable, as only one population contained green fluorescent protein (GFP) (Fig. 1d-f). We found that GFP-labelled cells crossed the interface; however, when comparing the dual and single cell migration assays, there was significantly less migration when both scaffolds were pre-loaded with cells $(\mathrm{p}<0.002)$, in which case the cells did not fully invade the adjacent scaffold. Therefore, the two cell populations remained largely distinct away from the interface on their respective sides but were well integrated at the interface (Fig. 1e-g).

\section{Bone-connective tissue interface}

ETIs can be designed to replicate specific tissue interfaces, such as the bone-connective tissue interface, which delineates two separate regimes (Fig. 2). This was achieved by culturing MC 3T3 E1 subclone 4 pre-osteoblasts on one subunit to confluency followed by differentiation into osteoblasts, which mineralized the scaffold. An opposing scaffold was also cultured until confluence with mouse NIH 3 T3 fibroblasts and interlocked with the calcified subunit (Fig. 2).

As osteoblasts deposit calcium and mineralize their extracellular matrix, there are different biochemical and physical environments of the two cell populations forming the bone-connective tissue ETI. Alkaline phosphatase and calcium staining revealed that the composite was comprised of two distinct regions away from the interface (Fig. 2 and Supplementary Fig. 3). We then probed the local cellular environments using scanning electron microscopy (SEM) and electron dispersion spectroscopy (EDS). SEM revealed two distinct surface topographies (Fig. 2c, f and 
Supplementary Fig. 4). On the bone component, a smooth coating was deposited on the scaffold (Fig. 2c, f and Supplementary Fig. 4). This coating was absent on the fibroblast side; instead, thick layers of cells were observed (Fig. 2c, f and Supplementary Fig. 4). EDS was employed to confirm that the smooth coating on the differentiated bone component was mineralization. The characteristic X-rays confirmed the presence of calcium $(\sim 3.69 \mathrm{keV})$ and phosphorous $(\sim 2.01 \mathrm{keV})$ on the bone scaffolds (Fig. 2g). These elements were not present to a detectable level on the fibroblast side (Fig. 2h, i). The interfacial region was also devoid of mineralization.

The separation of the mineralized and non-mineralized tissue resulted in two regions with different mechanical profiles (Fig. 2). All tissue interfaces in our bodies experience stress shielding when the mechanical environments of the constituents vary. The phenomenon of stress shielding is the difference in the stress applied to each body in a cohesive system. The composite is composed of materials with different elastic moduli; therefore, an effective Young's modulus can be obtained from the geometry, direction of applied force, and the moduli of the constituents (Fig. 2j). ETIs consisting of components with different mechanical profiles allows stress shielding biomaterials to be formed ${ }^{7,38,39}$ (Fig. 2 j and Supplementary Fig. 5). For example, two prototype geometries can be considered: two bricks are stacked on top of one another and a force is applied normal to the interface (series orientation) or parallel to it. The effective Young's modulus in the series arrangement was $13.8 \pm 3.3 \mathrm{kPa}$ which was significantly less than the parallel conformation with a value of $44.2 \pm 8.4(\mathrm{p}<0.001)$. In addition to macroscale compressions, atomic force microscopy was used to probe the local mechanics in both regions (Fig. 2k); the two stiffness regimes observed were comparable to the reported literature values for bone and fibroblast cells 46,47. The bone component had a Young's modulus of $115.0 \pm 8.2 \mathrm{kPa}$, whereas the fibroblast component had a significantly lower modulus of $3.9 \pm 0.3 \mathrm{kPa}(\mathrm{p}<0.001)$. Interestingly, there was 
an intermediate stiffness found in the interfacial region with a modulus of $21.7 \pm 1.7 \mathrm{kPa}$, as expected for a region composed of fibroblasts, undifferentiated pre-osteoblasts, and differentiated osteoblasts surrounded by their mineralized matrix. A cell migration assay from the fibroblast component onto the bone section demonstrated that cells intermixed at the interface and exhibited a $13 \%$ proliferation increase as in the previous experiment $(\mathrm{p}=0.971$, compared to the dual fibroblast migration assay), which is similar to the natural bone-connective tissue interface ${ }^{48,49}$ (Fig. 21, m).

\section{Mechanical characterization of ETIs}

Mechanical characterization is integral for designing tissue interfaces with specialized structure-function relationships ${ }^{49}$. Three separate mechanical characterizations are of particular interest for these interlocked composite materials: compression, tension, and shear (Fig. 3a-f). We examined scaffolds with and without added fibroblast cells in the blocks. Compression tests probe the bulk elastic properties of the two blocks in series, and the interface between blocks plays a minor role. It was found that the presence of cells had no significant impact on the apparent stiffness of the material: $24.4 \pm 1.3 \mathrm{kPa},(\mathrm{p}=0.68)(\mathrm{Fig} .3 \mathrm{~g})$, as intrinsic cell elasticity is relatively weak compared to that of the matrix material. Under tension, the mechanical response is dominated by the cohesiveness of the interfacial region. This can be seen clearly in Fig. 4e, which shows a progressively decreasing contact area between blocks as two blocks separate under the applied tension. In this case, the stress required to initiate separation of the two subunits was greater when fibroblasts were seeded on the scaffolds (cells: $1.56 \pm 0.1 \mathrm{kPa}$, control: $0.6 \pm 0.1 \mathrm{kPa}, \mathrm{p}<0.001$ ) (Fig. 3h), as cell-matrix and cell-cell adhesion both contribute to the mechanical integrity of the interfacial region. Applied shear provides a direct probe of interfacial cohesion and integrity. 
Controlled steady shear rate experiments were characterized by an initial reversible response, where stress $\sigma$ is found to be a sub-linear power law of applied strain $\varepsilon, \sigma \sim \varepsilon^{\mathrm{y}}$ with $\mathrm{y} \cong 3 / 4$, followed by subsequent strain softening (Fig. 3i). The lack of a linear stress-strain relationship at low strains is indicative of mechanics dominated by stick-slip events at the interface. For larger strains, eventually transient failure in the slip plane of the interface occurs, which is followed by a steady state friction response (Supplementary Fig. 6). Interestingly, the power-law stress-strain response was found to be reversible even after failure in the slip plane for both relatively slow and fast applied strain rates (Supplementary Fig. 6), which may serve as a valuable design feature for preventing the scaffolds from irreversible fracturing under large loads in situ. In shear conditions, the presence of fibroblast cells did not qualitatively change the stress-strain relationship; however, samples with cells were found to be somewhat more compliant (Fig. 3i). An interfacial region containing cells, which have a relatively lower intrinsic modulus, would be more compliant than an interface composed of pure matrix.

\section{In vivo implantation}

The potential applications of ETIs are not limited to in vitro studies of tissue interfaces; we foresee ETIs playing an essential role in biomaterial implant design. As a proof-of-concept experiment, we implanted the interlocked composite materials subcutaneously in an immunocompetent rat model for 4 weeks $^{5,6}$ (Fig. 4a). The subunits remained interlocked despite being placed under the skin (Fig. 4b). Histological analysis revealed that the cells invaded the peripheral regions of the biomaterials, and blood vessel formation was clearly observed (Fig. 4c-f and Supplementary Fig. 7) 5 . The invading fibroblasts from the surrounding animal tissue laid down their own collagen network within the cellulose-based scaffold (Fig. 4d, f). 


\section{DISCUSSION}

As demonstrated in this study, ETIs establish a novel approach for studying and implementing tissue interface models. Significant insights in matrix composition, mechanical loading, and cell organization have been gained from specific tissue interface models of entheses, such as the lack of transition and insertion zones at interfaces post-operations $1,2,50,3,7,24-27,45,49$, yet platforms to study tissue interfaces are lacking. We have shown that decellularized plant-derived scaffolds can be shaped into complementary interlocking subunits, and that cells can migrate across the interface between such subunits. In the absence of an existing cell population, such as an implanted biomaterial void of cells, this extensive cell migration and infiltration is usually desirable ${ }^{51}$. When a neighbouring cell population was present, migration across the interface was observed, yet the migrating cells did not fully invade the adjacent scaffold. This result is pertinent for tissue interfaces, as a uniform co-culture of cells does not replicate entheses in vivo. Two obstacles in entheses design are the achievement of adequate cell invasion and the creation of an appropriate transitional zone between segregated microenvironments ${ }^{52-54}$. The modular assembly with porous scaffolds enables cells from two distinct regions to form an intermediate zone at the interface between the subunits ${ }^{50}$.

We highlighted the specific example of creating a bone-connective tissue interface. Bone cells are surrounded by a mineralized extracellular network; however, this mineralization is absent in the connective tissue $\mathrm{e}^{1-3,24-27}$. A lack of integration of the two regions is a key factor in implant failure $^{2}$. We showed that cells migrated across the interface and that distinct mechanical environments were established. In this interfacial region between the two scaffolds, no significant mineralization was observed. In vivo, there is a similar zone in the enthesis known as the 
uncalcified avascular fibrocartilage zone, which is a non-mineralized, avascular region found between the fibrous connective tissue and the tidemark separating the hard and soft tissues ${ }^{48}$.

Several models have been developed in an attempt to recreate this interface; the approach consists of creating different environments within a single stratified material or two or more adjacent components ${ }^{1-3,24-27}$. Nevertheless, a universal "plug and play" system has not been established for engineering and designing models of interfaces.

Spatial organization and transitional zones within tissues and tissue interfaces are highly complex and integral to proper function; thus, the ability to combine them in a spatially controlled manner is essential. The modular assembly of components into a functional unit allows customizable designer interfaces to be formed. The choice of scaffold base material and interlocking format are important considerations when developing the artificial tissue enthesis. Here, we observed that the presence of cells increased the integration of the two subunits, as evidenced by tension experiments; however, no measurable effect was observed during the compression and rotation tests. A different dependence on cells would be plausible for other scenarios such as different cell types and/or different interlocking geometries (e.g. multi-post blocks).

The ETIs are also suitable for in vivo applications as well. It was found that the interlocked material did not disassemble in vivo. It is easy to speculate how these materials may be used to study and perhaps replace of regenerate tissue enthesis in the body. From a different angle, these materials also offer the ability to study complex systems and processes associated with tissue interface barriers, which can be found throughout the body, such as in the kidney, liver, intestines, and the brain. Significant advances have been made in microfluidics to study these tissue barriers; however, the focus is almost entirely on the fluid-barrier interface. By using ETIs in a 
combinatorial approach with the recent microfluidic advances, a deeper understanding of how flow across the barrier can affect cells of stratified tissues may be achieved ${ }^{55-57}$. The modular assembly approach of ETIs presented here ultimately provides an exciting new framework for the design of living tissues.

\section{MATERIALS AND METHODS}

\section{Scaffold production}

A Shapeoko $3 \mathrm{CNC}$ router with a $0.8 \mathrm{~mm}, 180^{\circ}$ drill bit was used to carve McIntosh Red apples (Canada Fancy) into arrays of complementary stud and anti-stud geometries. Cutting was performed at a speed of $1 \mathrm{~mm} / \mathrm{s}$. A Mandolin slicer was then used to cut the arrays of the subunits to their desired thickness. The stud piece consisted of a $5 \mathrm{~mm} \times 5 \mathrm{~mm} \times 2 \mathrm{~mm}$ base with a $2 \mathrm{~mm}$ cylindrical peg protruding from the center, and the anti-stud subunit had a $2 \mathrm{~mm}$ cylindrical hole in the center of the $5 \mathrm{~mm} \times 5 \mathrm{~mm} \times 2 \mathrm{~mm}$ base. As done previously, the samples were transferred to a $0.1 \%$ SDS solution and decellularized for $48 \mathrm{~h}$ while being shaken at $180 \mathrm{RPM}$, then were washed three times with $\mathrm{dH}_{2} \mathrm{O}$. Following the washes, the subunits were incubated in $100 \mathrm{mM}$ $\mathrm{CaCl}_{2}$ for $24 \mathrm{~h}$ and were then washed three times with $\mathrm{dH}_{2} \mathrm{O}$ to remove the salt residue. To sterilize the samples, a $70 \%$ ethanol incubation was performed, followed by three more wash cycles with $\mathrm{dH}_{2} \mathrm{O}$.

\section{Cell culture}

All cells were maintained at $37^{\circ} \mathrm{C}$ and $5 \% \mathrm{CO}_{2}$. NIH $3 \mathrm{~T} 3$ fibroblast cells were cultured in Dulbecco's Modified Eagle Medium - High Glucose (DMEM), supplemented with 10\% fetal bovine serum and $1 \%$ penicillin/streptomycin $(100 \mathrm{U} / \mathrm{mL}$ and $100 \mu \mathrm{g} / \mathrm{mL}$ respectively) (Hyclone 
Laboratories Inc.). Conversely, the MC 3T3 E1 Subclone 4 pre-osteoblast cells were culture in MEM-alpha supplemented with $10 \%$ fetal bovine serum and 1\% penicillin/streptomycin (100 $\mathrm{U} / \mathrm{mL}$ and $100 \mu \mathrm{g} / \mathrm{mL}$ respectively). In order to invoke differentiation of the pre-osteoblasts, $4 \mathrm{mM}$ inorganic phosphate (Sigma) and $50 \mu \mathrm{g} / \mathrm{mL}$ ascorbic acid (Sigma) were added. For sub-culturing, cells cultured on cell culture plates were trypsinized and resuspended in the appropriate medium. The cells were counted and centrifuged in order to separate the cells from the trypsin and the media. The supernatant was aspirated, and the pellet containing $5 \times 10^{4}$ cells was resuspended in fresh culture medium. The cells were seeded onto the biomaterial, and the cells were allowed to proliferate and invade the scaffold for 2 weeks prior to interlocking the complementary subunits. After 2 weeks, the subunits were manually clicked together using sterile tweezers by pressing down the stud subunit into the hole of the anti-stud piece. The culture media was replaced every day and the samples were transferred to new culture plates after 1 week of growth.

\section{Confocal microscopy}

The biomaterials were imaged with a Nikon TiE A1-R confocal microscope. The plant-derived scaffold was visualized with Calcofluor white staining (30 min, $1 \mu \mathrm{g} / \mathrm{mL}$, Sigma) and Congo Red (30 min, $0.1 \mu \mathrm{g} / \mathrm{mL}$, Sigma). Cell nuclei were stained with Hoechst 33342 (Invitrogen) (5 min incubation, $10 \mu \mathrm{g} / \mathrm{mL}$ ). Image (Fiji) was used to process the images; brightness/contrast settings were adjusted to maximize the fluorophore signal.

\section{Alkaline phosphatase staining}

Prior to fixation, the scaffolds were washed with PBS. They were then fixed for $90 \mathrm{~s}$ with $3.5 \%$ paraformaldehyde and then washed with wash buffer $(0.05 \%$ Tween in PBS). The BCIP-NBT 
SigmaFast ${ }^{\mathrm{TM}}$ ) tablets were dissolved in $\mathrm{dH}_{2} \mathrm{O}$ and staining and imaging were completed within 1

h. During the staining, the samples were kept in the dark and were monitored. Once the staining was complete, the samples were washed and photographed.

\section{Alizarin Red S staining}

Alizarin Red S (Sigma) was prepared by adding $1 \mathrm{~g}$ of the powder to $45 \mathrm{~mL}$ of $\mathrm{dH}_{2} \mathrm{O}$. The $\mathrm{pH}$ was then adjusted to 4.3 with $\mathrm{HCl}$ and $\mathrm{NaOH}$ before raising the volume to $50 \mathrm{~mL}$. Prior to staining, the samples were fixed as outlined above, except the duration of the fixation process was $>1 \mathrm{~h}$. The biomaterials were then washed with PBS. Calcium staining was performed with a $0.22 \mu \mathrm{m}$ filtered Alizarin Red S stain pH 4.3 at a concentration of $200 \mathrm{mg} / \mathrm{mL}$. The samples were submerged in the stain and incubated for 45 min. Following the calcium staining, the samples were thoroughly washed with $\mathrm{dH}_{2} \mathrm{O}$ until the colour ceased to run out of the samples. The samples imaged shortly afterwards.

\section{Cell migration assays}

The confocal images of the GFP 3 T3 cells were thresholded using the ImageJ (Fiji) adaptive threshold plugin, and the analyze particles plugin was used to measure the proliferation area on the adjacent scaffold. The data was normalized to the average scaffold area. The values presented are mean values \pm the standard error of the mean (s.e.m.).

\section{Compression and tension testing}

The compressive Young's modulus and the maximum tensile stress were measured using the compression and tension modes of a custom built dynamic mechanical analysis (DMA) device and 
LabVIEW. During the compression tests, the material was compressed to a $10 \%$ strain, at a strain rate of $50 \mu \mathrm{m} / \mathrm{s}$. The force-displacement curves were converted to stress-strain curves, and they were fitted in Origin 8.5 to calculate the Young's modulus. Likewise, the tensile measurements involved recording the minimum force required to separate the subunits at the same motor speed).

\section{Scanning Electron Microscopy}

Samples were fixed with $3.5 \%$ paraformaldehyde for $48 \mathrm{~h}$ and washed with PBS. The samples were serially dehydrated with ethanol as indicated in Intech Open's sample preparation guide ${ }^{58}$. The samples were dried with a samdri-PVT-3D critical point drier, and then gold-coated in a Hitachi E-1010 ion sputter. Scanning electron microscopy (SEM) and energy dispersive X-ray spectroscopy (EDS) were performed on a JOEL JSM-7500F field emission SEM at the Centre for Advanced Materials Research (CAMaR) at the University of Ottawa. The SEM images were recorded at $3 \mathrm{kV}$, and the EDS spectra were recorded at $15 \mathrm{kV}$.

\section{Atomic Force Microscopy}

A Nanowizard II atomic force microscope (AFM) (JPK Instruments, Germany) was used to determine the substrate elasticity in all experiments. PNP-TR-50 cantilevers were used for each measurement and had an experimentally determined spring constant of $63.5 \pm 7.2 \mathrm{mN} / \mathrm{m}$. Forceindentation curves were acquired on substrates at $546 \mathrm{~Hz}$ with a set point of $1.0 \mathrm{nN}$. Substrate elasticity was calculated by fitting the force curves to the Sneddon-Hertz model for a conical indenter for shallow $200 \mathrm{~nm}$ indentations, assuming a Poisson ratio of 0.5 (PUNIAS 3D Software). For each substrate, $\mathrm{N}=3$ plates were prepared and 10 force curves were acquired at 25 random locations on the substrate for a total of 250 force curves. 


\section{Rheometry}

An MCR 301 Anton Paar Rheometer was used to determine the shear properties of the materials. The samples were measured using a parallel-plate geometry with a circular $12 \mathrm{~mm}$ diameter. The samples were glued to the surface using Permabond Instant Adhesive 102 Medium Viscosity General Purpose Glue: a thin layer of the glue was evenly spread on the surface and the samples were gently pressed onto it and were incubated for 10 minutes prior to experimentation. Repeat runs of frequency sweeps revealed the samples were well adhered and did not slip. The samples were hydrated with $\mathrm{CO}_{2}$ independent media (25 mM HEPES) to prevent the samples from drying and resulting changes to scaffold mechanical properties.

\section{Animal surgeries and implantation}

The subcutaneous implantation protocol was similar to what we have used previously. Briefly, immunocompetent rats were anesthetized using 2\% Isoflurane USP-PPC (Pharmaceutical partners of Canada, Richmond, ON, Canada). ENDURE 400 Scrub-Stat4 Surgical Scrub (chlorhexidine gluconate, 4\% solution; Ecolab Inc., Minnesota, USA) and Soluprep (2\% w/v chlorhexidine and $70 \%$ v/v isopropyl alcohol; 3M Canada, London, ON, Canada) were used to prepare the shaved dorsal ventral area. Three $8 \mathrm{~mm}$ incisions were cut on the dorsal section of each rat, and a combined barren scaffold was placed in each incision. The incisions were then sutured using Surgipro II monofilament polypropylene 6-0 (Covidien, Massachusetts, USA). Transdermal bupivacaine 2\% (as monohydrate; Chiron Compounding Pharmacy Inc., Guelph, ON, Canada) was topically applied to the surgery sites to prevent infection, and buprenorphine $(0.03 \mathrm{mg} / \mathrm{ml}$; Chiron 
Compounding Pharmacy Inc. Guelph, ON, Canada) was administrated to alleviate pain. Animals were monitored and the sutures were removed after one week.

\section{Scaffold resections}

At 4 weeks post-implantation, the mice were euthanized using $\mathrm{CO}_{2}$ inhalation and exsanguination via heart dissection. The dorsal skin was carefully resected and fixed in $10 \%$ formalin for at $72 \mathrm{~h}$. The samples were then kept in $70 \%$ ethanol before being embedded in paraffin by the PALM Histology Core Facility of the University of Ottawa.

\section{Histology}

Serial $4 \mu \mathrm{m}$ thick microtome sections starting at the edge of the cellulose scaffolds were cut at 100 $\mu \mathrm{m}$ levels. The sections were stained with Hematoxylin and Eosin (H\&E) and Masson's Trichrome. For immunohistochemistry, the sections stained with Rabbit anti-CD45 (Abcam) or Rabbit anti-CD31 (Novus) were pre-treated using heat mediated antigen retrieval with a citrate buffer ( $\mathrm{pH}$ 6.0, epitope retrieval solution 1) for 20 minutes. The sections were then incubated using a 1:1600 dilution (CD45) or 1:100 (CD31) for 30 minutes at room temperature and detected using an HRP conjugated compact polymer system. The slides were then stained with DAB as the chromogen, counterstained with Hematoxylin, mounted, and cover slipped.

\section{Statistical analysis}

For multiple samples, one-way and two-way ANOVA tests were used to assess the statistical differences for samples with one or two factors respectively. The Tukey post hoc analysis was used to determine the value of the statistical difference between the individual samples. In the cases where only two sets of data were compared, the Student's t-tests was used. All values 
presented are the mean \pm the standard error of the mean (s.e.m.). Statistical significance refers to $\mathrm{P}<0.05$.

\section{ACKNOWLEDGEMENTS}

This work was supported by individual National Sciences and Engineering Research Council Discovery Grants awarded to AEP and JLH.

\section{AUTHOR CONTRIBUTIONS}

RJH oversaw all experimental protocols and fabricated the scaffolds, performed cell culture, microscopy and analysis. MLL performed cell culture. MLL and RJH performed the surgical procedures. RJH and JLH performed rheological analyses. AEP conceived, directed and managed the project. All authors reviewed the data and prepared the manuscript for publication.

\section{COMPETING INTERESTS}

RJH, MLL and AEP are inventors of multiple patents regarding the creation and use of plantderived cellulose biomaterials. They are also former or current employees of Spiderwort Inc. which is leading the clinical translation of these biomaterials. JLH declares no competing interests.

\section{REFERENCES}

1. Boys, A. J., McCorry, M. C., Rodeo, S., Bonassar, L. J. \& Estroff, L. A. Next generation tissue engineering of orthopedic soft tissue-to-bone interfaces. MRS Commun. 7, 289-308 (2017).

2. Patel, S. et al. Integrating Soft and Hard Tissues Via Interface Tissue Engineering. $J$ 
Orthop Res. 36, 6072-6078 (2018).

3. Harris, E. et al. Biofabrication of soft tissue templates for engineering the bone-ligament interface. Biotechnol. Bioeng. 114, 2400-2411 (2017).

4. Modulevsky, D. J., Lefebvre, C., Haase, K., Al-Rekabi, Z. \& Pelling, A. E. Apple derived cellulose scaffolds for 3D mammalian cell culture. PLoS One 9, (2014).

5. Modulevsky, D. J., Cuerrier, C. M. \& Pelling, A. E. Biocompatibility of Subcutaneously Implanted Plant-Derived Cellulose Biomaterials. PLoS One 11, 1-19 (2016).

6. Hickey, R. J., Modulevsky, D. J., Cuerrier, C. M. \& Pelling, A. E. Customizing the Shape and Microenvironment Biochemistry of Biocompatible Macroscopic Plant-Derived Cellulose Scaffolds. ACS Biomater. Sci. Eng. 4, 3726-3736 (2018).

7. Bayrak, E. \& Yilgor Huri, P. Engineering Musculoskeletal Tissue Interfaces. Front. Mater. 5, 24 (2018).

8. Place, E. S., Evans, N. D. \& Stevens, M. M. Complexity in biomaterials for tissue engineering. Nat. Mater. 8, 457-470 (2009).

9. Das, R. K. \& Zouani, O. F. A review of the effects of the cell environment physicochemical nanoarchitecture on stem cell commitment. Biomaterials 35, 5278-5293 (2014).

10. Skylar-Scott, M. A. et al. Biomanufacturing of organ-specific tissues with high cellular density and embedded vascular channels. Sci. Adv. 5, eaaw2459 (2019).

11. Mironov, V. et al. Organ printing: tissue spheroids as building blocks. Biomaterials 30, 2164-74 (2009). 
12. Valentin, T. M. et al. 3D printed self-adhesive PEGDA-PAA hydrogels as modular components for soft actuators and microfluidics. Polym. Chem. 10, 2015-2028 (2019).

13. Grigoryan, B. et al. Multivascular networks and functional intravascular topologies within biocompatible hydrogels. Science 364, 458-464 (2019).

14. Marti-Figueroa, C. R. \& Ashton, R. S. The case for applying tissue engineering methodologies to instruct human organoid morphogenesis. Acta Biomater. 54, 35-44 (2017).

15. Lee, J., Jung, H., Park, N., Park, S. H. \& Ju, J. H. Induced Osteogenesis in Plants Decellularized Scaffolds. Sci. Rep. 9, 1-10 (2019).

16. Toker, M. et al. Decellularization and characterization of leek: a potential cellulose-based biomaterial. Cellulose 27, 7331-7348 (2020).

17. Gershlak, J. et al. Crossing kingdoms: Using decellularized plants as perfusable tissue engineering scaffolds. Biomaterials 125, 13-22 (2017).

18. Fontana, G. et al. Biofunctionalized Plants as Diverse Biomaterials for Human Cell Culture. Adv. Healthc. Mater. 1601225 (2017). doi:10.1002/adhm.201601225

19. Phan, N. V., Wright, T., Rahman, M. M., Xu, J. \& Coburn, J. M. In Vitro Biocompatibility of Decellularized Cultured Plant Cell-Derived Matrices. ACS Biomater. Sci. Eng. 6, 822-832 (2020).

20. Robbins, E. R., Pins, G. D., Laflamme, M. A. \& Gaudette, G. R. Creation of a contractile biomaterial from a decellularized spinach leaf without ECM protein coating: An in vitro study. J. Biomed. Mater. Res. - Part A 108, 2123-2132 (2020). 
21. Contessi Negrini, N., Toffoletto, N., Farè, S. \& Altomare, L. Plant Tissues as 3D Natural Scaffolds for Adipose, Bone and Tendon Tissue Regeneration. Front. Bioeng. Biotechnol. 8, 1-15 (2020).

22. Iravani, S. \& Varma, R. S. Plants and plant-based polymers as scaffolds for tissue engineering. Green Chem. 21, 4839-4867 (2019).

23. Cheng, Y.-W., Shiwarski, D. J., Ball, R. L., Whitehead, K. A. \& Feinberg, A. W. Engineering Aligned Skeletal Muscle Tissue Using Decellularized Plant-Derived Scaffolds. ACS Biomater. Sci. Eng. 6, 3046-3054 (2020).

24. Atesok, K. et al. Multilayer scaffolds in orthopaedic tissue engineering. Knee Surgery, Sport. Traumatol. Arthrosc. 24, 2365-2373 (2016).

25. Lu, H. H., Subramony, S. D., Boushell, M. K. \& Zhang, X. Tissue engineering strategies for the regeneration of orthopedic interfaces. Ann. Biomed. Eng. 38, 2142-2154 (2010).

26. Allan, K. S., Pilliar, R. M., Wang, J., Grynpas, M. D. \& Kandel, R. A. Formation of biphasic constructs containing cartilage with a calcified zone interface. Tissue Eng. 13, 167-177 (2007).

27. Alhadlaq, A. \& Mao, J. J. Tissue-engineered osteochondral constructs in the shape of an articular condyle. J. Bone Jt. Surg. - Ser. A 87, 936-944 (2005).

28. Liu, K., Mihaila, S. M., Rowan, A., Oosterwijk, E. \& Kouwer, P. H. J. Synthetic Extracellular Matrices with Nonlinear Elasticity Regulate Cellular Organization. Biomacromolecules 20, 826-834 (2019).

29. Toyjanova, J. et al. 3D Viscoelastic traction force microscopy. Soft Matter 10, 8095-8106 
(2014).

30. Rens, E. G. \& Merks, R. M. H. Cell Shape and Durotaxis Follow from Mechanical CellSubstrate Reciprocity and Focal Adhesion Dynamics: A Unifying Mathematical Model. (2019).

31. Hwang, J. H. et al. Artificial cellular nano-environment composed of collagen-based nanofilm promotes osteogenic differentiation of mesenchymal stem cells. Acta Biomater. 86, 247-256 (2019).

32. Gasiorowski, J. Z., Murphy, C. J. \& Nealey, P. F. Biophysical Cues and Cell Behavior: The Big Impact of Little Things. Annu. Rev. Biomed. Eng. 15, 155-176 (2013).

33. Entezari, A., Swain, M. V., Gooding, J. J., Roohani, I. \& Li, Q. A modular design strategy to integrate mechanotransduction concepts in scaffold-based bone tissue engineering. Acta Biomater. 118, 100-112 (2020).

34. Li, L., Eyckmans, J. \& Chen, C. S. Designer biomaterials for mechanobiology. Nat. Mater. 16, 1164-1168 (2017).

35. Chighizola, M. et al. Mechanotransduction in neuronal cell development and functioning. Biophys. Rev. 11, 701-720 (2019).

36. Naqvi, S. M. \& McNamara, L. M. Stem cell mechanobiology and the role of biomaterials in governing mechanotransduction and matrix production for tissue regeneration. Front. Bioeng. Biotechnol. 8, 1375 (2020).

37. Duval, K. et al. Modeling physiological events in 2D vs. 3D cell culture. Physiology 32, 266-277 (2017). 
38. Weinans, H. \& Huiskes, R. I. K. The Relationship Between Stress Shielding and Bone Resorption Around Total Hip Stems and the Effects of Flexible Materials. Clin. Orthop. Relat. Res. 274, 124-134 (1992).

39. Pakvis, D. F. M., Heesterbeek, P. J. C., Severens, M. \& Spruit, M. Cancellous and cortical bone mineral density around an elastic press-fit socket in total hip arthroplasty: A prospective 2-year follow-up study using quantitative CT BMD measurements in 25 patients. Acta Orthop. 87, 583-588 (2016).

40. Jaalouk, D. E. \& Lammerding, J. Mechanotransduction gone awry. Nat. Rev. Mol. Cell Biol. 10, 63-73 (2009).

41. Shaw, H. M. \& Benjamin, M. Structure-function relationships of entheses in relation to mechanical load and exercise: Review. Scand. J. Med. Sci. Sport. 17, 303-315 (2007).

42. Boys, A. J. et al. Understanding the Stiff-to-Compliant Transition of the Meniscal Attachments by Spatial Correlation of Composition, Structure, and Mechanics. ACS Appl. Mater. Interfaces 11, 26559-26570 (2019).

43. Cambré, I. et al. Mechanical strain determines the site-specific localization of inflammation and tissue damage in arthritis. Nat. Commun. 9, 1-14 (2018).

44. Deymier, A. C. et al. The multiscale structural and mechanical effects of mouse supraspinatus muscle unloading on the mature enthesis. Acta Biomater. 83, 302-313 (2019).

45. Wang, I.-N. E. et al. Role of Osteoblast-Fibroblast Interactions in the Formation of the Ligament-to-Bone Interface. J. Orthop. Res. 25, 16091620 (2007). 
46. Takai, E., Costa, K. D., Shaheen, A., Hung, C. T. \& Guo, X. E. Osteoblast Elastic

Modulus Measured by Atomic Force Microscopy Is Substrate Dependent. Ann. Biomed.

Eng. 33, 963-971 (2005).

47. Ford, A. J. \& Rajagopalan, P. Measuring Cytoplasmic Stiffness of Fibroblasts as a

Function of Location and Substrate Rigidity Using Atomic Force Microscopy. ACS

Biomater. Sci. Eng. 4, 3974-3982 (2018).

48. Apostolakos, J. et al. The enthesis: A review of the tendon-to-bone insertion. Muscles.

Ligaments Tendons J. 4, 333-342 (2014).

49. Lu, H. H. \& Thomopoulos, S. Functional Attachment of Soft Tissues to Bone:

Development, Healing, and Tissue Engineering. Annu. Rev. Biomed. Eng. 15, 201-226

(2013).

50. Yang, P. J. \& Temenoff, J. S. Engineering orthopedic tissue interfaces. Tissue Eng. - Part

B Rev. 15, 127-141 (2009).

51. Joly, P. et al. Geometry-Driven Cell Organization Determines Tissue Growths in Scaffold Pores: Consequences for Fibronectin Organization. PLoS One 8, 1-11 (2013).

52. Chow, L. W. \& Fischer, J. F. Creating biomaterials with spatially organized functionality. Exp. Biol. Med. (Maywood). 241, 1025-32 (2016).

53. Li, Q., Ma, L. \& Gao, C. Biomaterials for in situ tissue regeneration: Development and perspectives. J. Mater. Chem. B 3, 8921-8938 (2015).

54. Sakaguchi, K., Shimizu, T. \& Okano, T. Construction of three-dimensional vascularized cardiac tissue with cell sheet engineering. J. Control. Release 205, 83-88 (2015). 
55. Thoo, L., Noti, M. \& Krebs, P. Keep calm: the intestinal barrier at the interface of peace and war. Cell Death Dis. 10, (2019).

56. Yeste, J., Illa, X., Alvarez, M. \& Villa, R. Engineering and monitoring cellular barrier models. J. Biol. Eng. 12, 1-19 (2018).

57. Vera, D. et al. Engineering Tissue Barrier Models on Hydrogel Microfluidic Platforms. ACS Appl. Mater. Interfaces 13, 13920-13933 (2021).

58. Das Murtey, M. \& Ramasamy, P. Sample Preparations for Scanning Electron Microscopy - Life Sciences. in Modern Electron Microscopy in Physical and Life Sciences (eds. Janecek, M. \& Kral, R.) 1, 13 (Intech Open, 2016). 

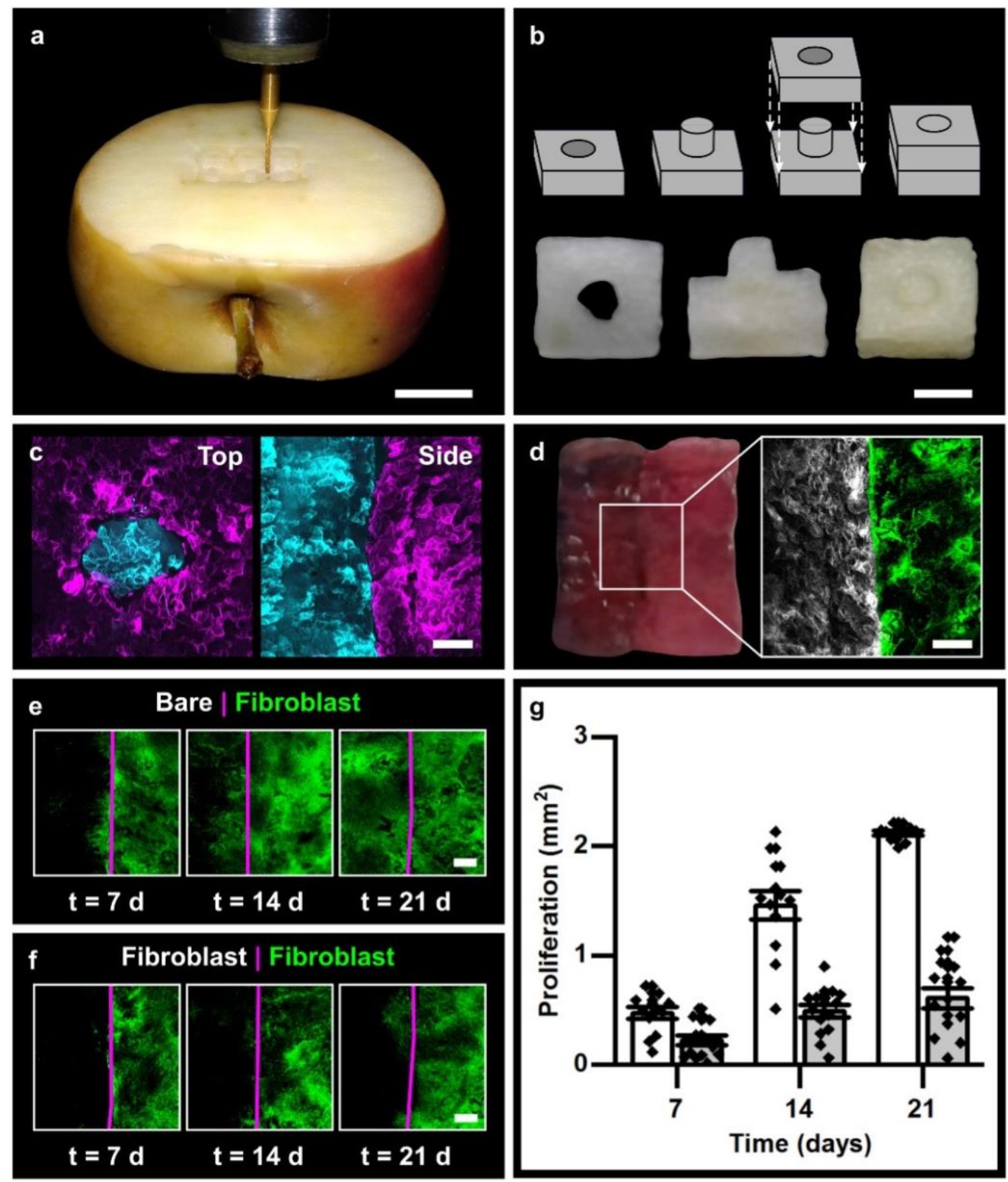

Fig. 1: Interlocked biomaterials for ETI formation. (a) A CNC router was used to carve the interlocking materials. Scale $=15 \mathrm{~mm}$. (b) Geometry of the single stud and anti-stud interlocked units as well as the decellularized subunits and the combined entity. Scale $=2.5 \mathrm{~mm}$. (c) Maximum z-projected confocal image of the top view and side view of the interlocked unit with one subunit stained with Calcofluor white (cyan) and the other stained with Congo Red (magenta), scale $=500$ $\mu \mathrm{m}$. (d) Tissue interface formation: left $=$ side view of interlocked unit, right $=$ maximum $\mathrm{z}$ projected confocal image of the two cell populations. White $=$ Hoechst 33342 stained nuclei of NIH 3 T3 cells, green $=$ GFP NIH 3T3 cells. Scale $=500 \mu \mathrm{m}$. Fibroblast cell (green) migration across the interface (magenta) of the interlocked unit with (e) one subunit preloaded with cells and the other void of cells, and (f) both loaded with cells (only one population transfected with GFP). Scale $=500 \mu \mathrm{m}$. (g) Quantification of cell migration across the interface. White $=$ bare $\mid$ fibroblast, grey = fibroblast $\mid$ fibroblast, $\mathrm{N}=12$. 

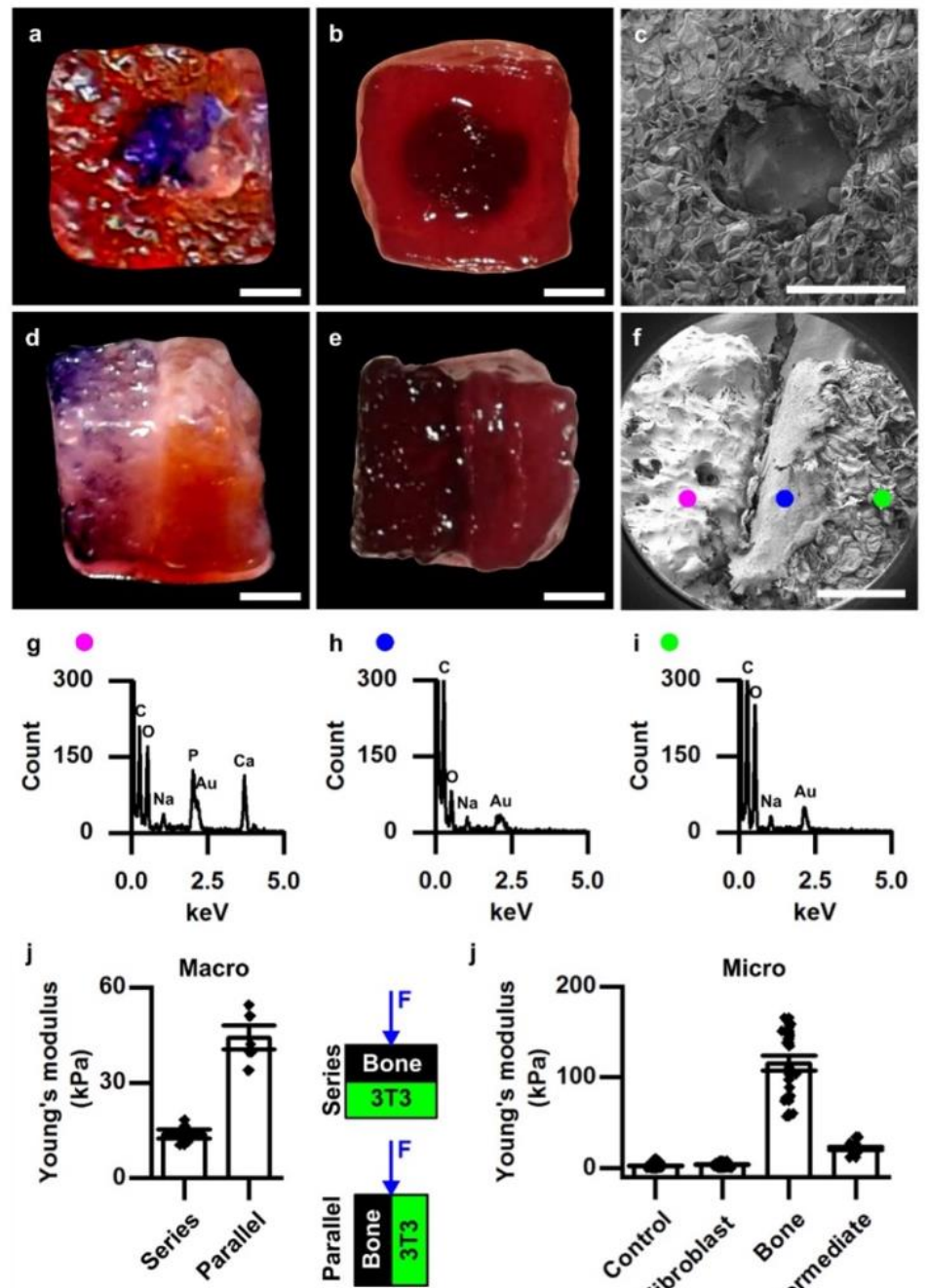

j
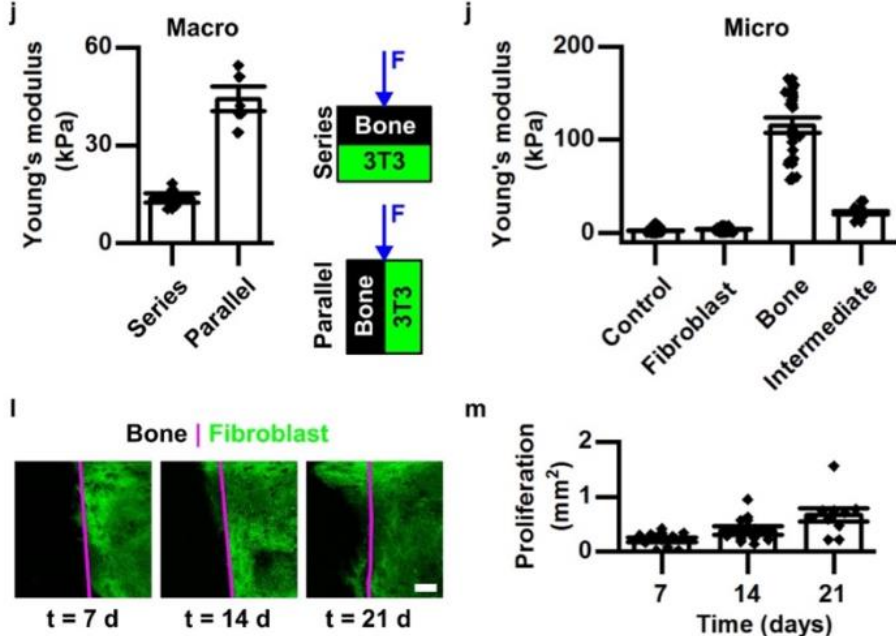

m

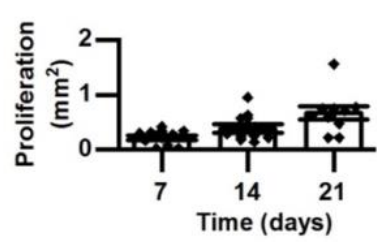

Fig. 2: Bone-connective tissue ETI. (a) Top view of scaffolds stained to reveal alkaline phosphate. Purple $=$ positive for alkaline phosphatase activity, orange/red $=$ negative. (b) Top view of scaffolds stained for the presence of calcium staining. Deep red $=$ calcified scaffold, light red $=$ non-calcified. (c) Top view of SEM of bone-connective tissue ETI with smooth mineralized peg inserted into the non-mineralized fibroblast covered subunit. Corresponding side views of the scaffolds stained for (d) alkaline phosphatase and (e) calcium. (f) Corresponding side view of the scaffold imaged with. Scale bar (a-f) $=1 \mathrm{~mm}$. (g-i) EDS chemical analysis of the corresponding regions (magenta $=$ bone subunit, blue $=$ fibroblast covered subunit adjacent to the interface, green = fibroblast covered subunit). (j) Compressive moduli of the bone-fibroblast ETI compressed in the series and parallel directions, $\mathrm{N}=5$. (k) Local mechanical probing: nanoindentations with AFM. (l) Fibroblast cell (GFP NIH 3T3) migration across the bone-connective tissue ETI, scale = $500 \mu \mathrm{m}$. (m) Quantification of cell migration, $\mathrm{N}=10$. 


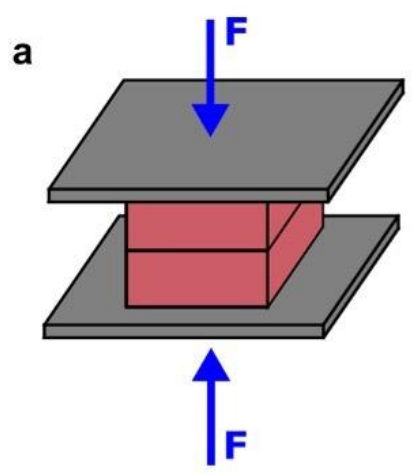

Compression
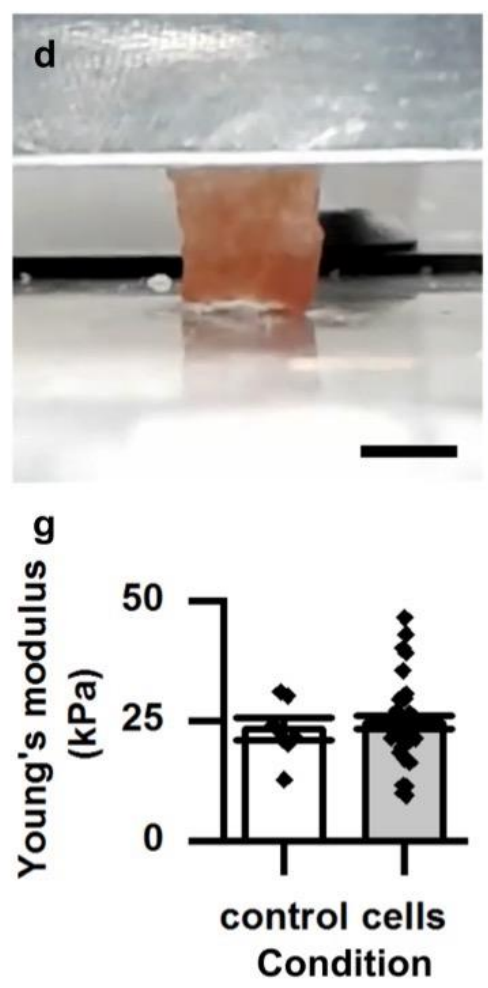

b

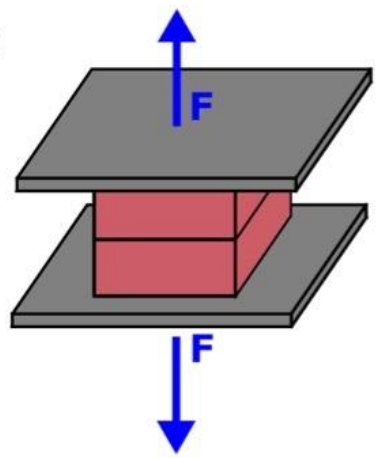

Tension

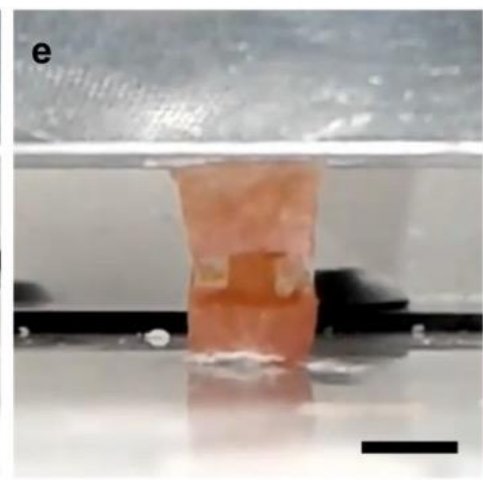

h

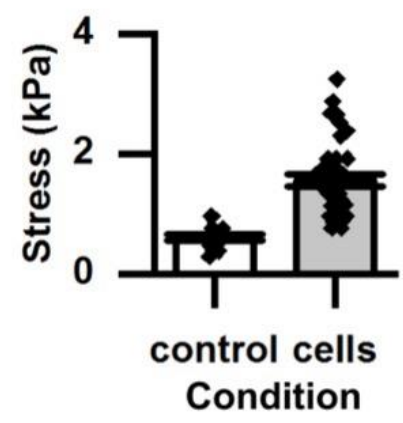

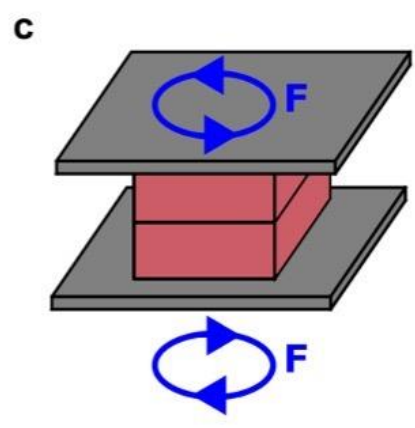

Shear

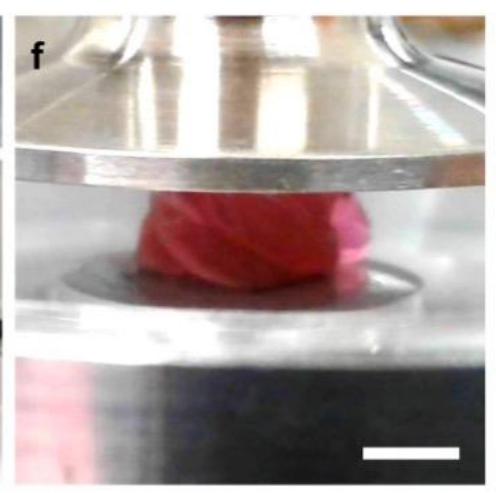

i

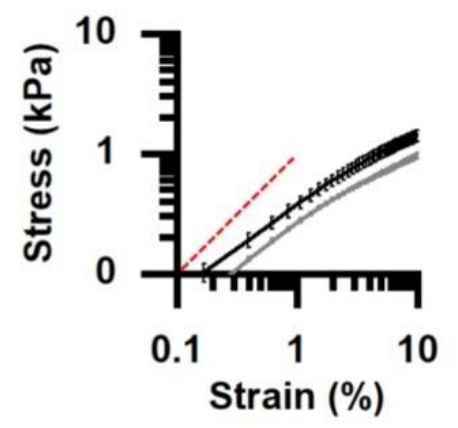

Fig. 3. Mechanical testing of ETIs. (a-c) Cartoon illustrations of the three classes of mechanical tests that were performed: compression, tension, and shear. (d-f) Interlocked ETIs under compressive, tensile, and shear stresses. Scale $=4 \mathrm{~mm}$. (g) Compressive Young's modulus with $(\mathrm{N}=36)$ and without fibroblast cells $(\mathrm{N}=7)$ with cells. (h) Maximum tensile stress prior to subunit separation with $(\mathrm{N}=39)$ and without fibroblast cells $(\mathrm{N}=15)$. (i) Shear stress $\sigma$ vs strain $\varepsilon$ in a controlled steady shear rate experiment at a strain rate of $0.01 \mathrm{~s}^{-1}, \mathrm{~N}=3$. Black $=$ without cells, grey $=$ with cells. The red dashed line is provided as a guide for the linear regime. Power-law behavior is observed at low strains: $\sigma \sim \varepsilon^{\mathrm{y}}$ for $\mathrm{y}=0.74 \pm 0.02$ without cells and $\mathrm{y}=0.72 \pm 0.02$ with cells. 
bioRxiv preprint doi: https://doi.org/10.1101/2020.11.06.371278; this version posted June 14, 2021. The copyright holder for this preprint (which was not certified by peer review) is the author/funder, who has granted bioRxiv a license to display the preprint in perpetuity. It is made available under aCC-BY-NC-ND 4.0 International license.
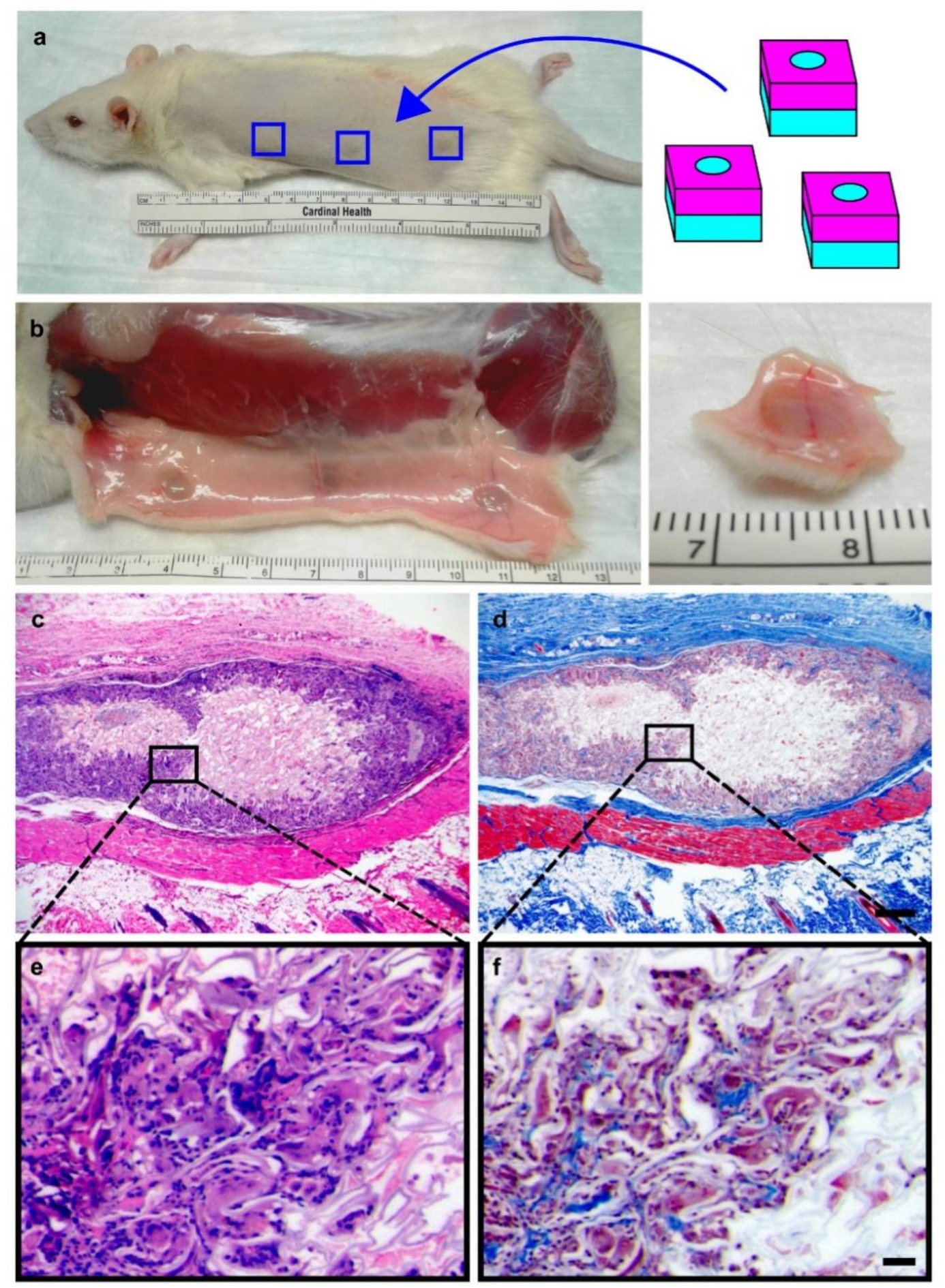

Fig. 4. In vivo implantation of ETIs. (a) Schematic showing the location of three subcutaneous implanted ETIs in a rat model. (b) ETI sample resection showing the subunits remained interlocked and underwent compression after 4 weeks. (c, e) H\&E staining revealing substantial of the cell invasion in the outer regions of the interlocked biomaterial. (d, f) Masson's Trichrome staining showing collagen deposition (blue) on the scaffold. Scale $(\mathbf{c}, \mathbf{d})=500 \mu \mathrm{m},(\mathbf{e}, \mathbf{f})=50 \mu \mathrm{m}$. 